\title{
A CHARACTERIZATION OF CLOSED IMAGES OF METRIC SPACES
}

\author{
L. FOGED
}

\begin{abstract}
We prove that a regular topological space is the image of a metric space under a closed mapping if and only if it is a Fréchet space with a $\sigma$-hereditarily closure-preserving $k$-network.
\end{abstract}

An internal characterization for topological spaces which are images of metric spaces under closed continuous functions, popularly called Lašnev spaces, has been given by Lašnev [6]. Here we present another characterization in terms of the $k$-networks introduced implicitly by Michael [8]: A collection $\mathscr{P}$ of closed subsets of a topological space $X$ is said to be a $k$-network for $X$ if, given any open set $U$ and any compact set $\overline{K \subset U}$, there is a finite subcollection $\mathscr{P}^{*}$ of $\mathscr{P}$ so that $K \subset \cup \mathscr{P} * \subset U$.

A family $\left\{A_{\alpha}: \alpha \in I\right\}$ of subsets of a space $X$ is said to be hereditarily closurepreserving if

$$
\bigcup_{\alpha \in J} \overline{B_{\alpha}}=\overline{\bigcup_{\alpha \in J} B_{\alpha}}
$$

whenever $J \subset I$ and $B_{\alpha} \subset A_{\alpha}$ for each $\alpha \in J$. A $\sigma$-hereditarily closure-preserving collection is a collection that is the union of countably many hereditarily closurepreserving families.

A topological space $X$ is a Fréchet space if, for every $A \subset X$, we have $\bar{A}=\{x \in X$ : $x$ is the limit of a convergent sequence in $A$ \}. All spaces are Hausdorff.

Our result is the following

THEOREM 1. A Hausdorff space $X$ is a Lašnev space if and only if $X$ is a Fréchet space with a $\sigma$-hereditarily closure-preserving $k$-network.

PROOF OF THE NECESSITY. It is known that closed mappings preserve the Fréchet property [1]. Let $M$ be a metric space, $f: M \rightarrow X$ a closed, continuous surjection, and $\mathscr{B}$ a $\sigma$-discrete base for $M$. Then one easily verifies that $\{f(\bar{B}): B \in \mathscr{B}\}$ is $\sigma$-hereditarily closure-preserving and, since every compact subset of $X$ is the image under $f$ of a compact subset of $M[7$, Corollary 1.2], that $\{f(\bar{B}): B \in \mathscr{B}\}$ is a $k$-network for $X$.

Before continuing with the proof of the sufficiency, we remark that if one removes the requirement that $k$-networks consist of closed sets, then a Lašnev space $X$ has a $k$-network which is the union of countably many hereditarily closurepreserving and point-finite collections. Indeed, let $\mathscr{P}=\bigcup_{n \in \mathbf{N}} \mathscr{P}_{n}$ be a $\sigma$-hereditarily

Received by the editors August 16, 1984 and, in revised form, November 20, 1984.

1980 Mathematics Subject Classification. Primary 54C10.

(1985 American Mathematical Society $0002-9939 / 85 \$ 1.00+\$ .25$ per page 
closure-preserving $k$-network of closed sets, and let $D_{n}=\left\{x \in X: \mathscr{P}_{n}\right.$ is not locally finite at $x\}$. Then $D_{n}$ is closed and discrete, for if $\left\{x_{k}: k \in \mathbf{N}\right\} \subset D_{n}$ then, because $\mathscr{P}_{n}$ is not point-finite at $x_{k}$, we can inductively choose $P_{k} \in \mathscr{P}_{n} \backslash\left\{P_{j}: j<k\right\}$ so that $x_{k} \in P_{k}$. The hereditarily closure-preserving property implies that $\left\{x_{k}: k \in \mathbf{N}\right\}$ is closed and thus, as $X$ is Fréchet, that $D_{n}$ is closed and discrete. It is now easy to see that $\mathscr{P}_{n}^{\prime}=\left\{P \backslash D_{n}: P \in \mathscr{P}_{n}\right\} \cup\left\{\{x\}: x \in D_{n}\right\}$ is point-finite, hereditarily closurepreserving and that $\cup_{n \in \mathbf{N}} \mathscr{P}_{n}^{\prime}$ satisfies the $k$-network property. This answers a question of [5].

LEMMA 2. If $\mathscr{R}$ is a hereditarily closure-preserving family of closed subsets of a Fréchet space $X$, then the collection $\left\{\cap \mathscr{R}^{*}: \mathscr{R}^{*}\right.$ is a finite subfamily of $\left.\mathscr{R}\right\}$ is also hereditarily closure-preserving.

Proof. If not, there is a collection $\left\{\mathscr{R}_{\alpha}: \alpha \in I\right\}$ of finite subfamilies of $\mathscr{R}$ and $G_{\alpha} \subset \cap \mathscr{R}_{\alpha}$ for $\alpha \in I$ so that $\bigcup_{\alpha \in I} \bar{G}_{\alpha}$ is not closed. Consequently one can find a sequence $\left\{z_{n}: n \in \mathbf{N}\right\}$ converging to an $x \in X \backslash \cup_{\alpha \in I} \bar{G}_{\alpha}$. We take $\alpha(n) \in I$ so that $z_{n} \in \overline{G_{\alpha(n)}}$ and, by choosing a subsequence, we may assume that the $\alpha(n)$ 's are distinct. It is possible to select an infinite $\{n(m): m \in \mathbf{N}\} \subset \mathbf{N}$ so that

$$
\mathscr{R}_{\alpha(n(m))} \backslash \bigcup_{k<m} \mathscr{R}_{\alpha(n(k))} \neq \varnothing .
$$

Picking $R_{m} \in \mathscr{R}_{\alpha(n(m))} \backslash \bigcup_{k<m} \mathscr{R}_{\alpha(n(k))}$ makes the $R_{m}$ 's distinct, and thus the fact that $z_{n(m)} \in R_{m}$, taken with the hereditarily closure-preserving property of $\mathscr{R}$, contradicts the convergence of $\left\{z_{n(m)}: m \in \mathbf{N}\right\}$.

LEMMA 3 [6]. If $\mathscr{R}$ is a hereditarily closure-preserving family in a space $X$ and $\left\{z_{n}\right.$ : $n \in \mathbf{N}\}$ is a sequence in $X \backslash\{x\}$ converging to $x$, then there is an $M \in \mathbf{N}$ so that $\left\{z_{n}\right.$ : $n \geqslant M\} \cap R \neq \varnothing$ for only finitely many $R \in \mathscr{R}$.

LEMMA 4. Let $X$ be a Fréchet space with a $\sigma$-hereditarily closure-preserving $k$-network $\mathscr{P}=\bigcup_{m \in \mathbf{N}} \mathscr{P}_{m}$ with $\mathscr{P}_{m} \subset \mathscr{P}_{m+1}$. If $U$ is an open set and $Z=\left\{z_{n}: n \in \mathbf{N}\right\} a$ sequence converging to $x \in U \backslash Z$, then there is an $N \in \mathbf{N}$ so that $Z$ is eventually in int $\bigcup\left\{P \in \mathscr{P}_{N}: P \subset U\right\}$.

Proof. For each $m \in \mathbf{N}$, let $\mathscr{P}_{m}^{*}=\left\{P \in \mathscr{P}_{m}: P \subset U\right\}$. If no $N \in \mathbf{N}$ satisfies the conclusion of Lemma 4 , then we may select a subsequence $\left\{z_{n(m)}: m \in \mathbf{N}\right\}$ so that $z_{n(m)} \in U \backslash$ int $\cup \mathscr{P}_{m}^{*}$. Because $X$ is Fréchet, there is a sequence $\left\{z_{n(m)}^{k}: k \in \mathbf{N}\right\}$ in $U \backslash \bigcup \mathscr{P}_{m}^{*}$ converging to $z_{n(m)}$. Now $x \in \overline{\left\{z_{n(m)}^{k}: m, k \in \mathbf{N}\right\}}$, so using the Fréchet property, the hypothesis that $x \notin Z$, and the Hausdorff property we can choose a sequence $Z^{\prime}$ of the form $\left\{z_{n(m(j))}^{k(j)}: j \in \mathbf{N}\right\}$, with $m(j)<m(j+1)$, that converges to $x$. Then there is an $M \in \mathbf{N}$ so that $Z^{\prime}$ is eventually in $\cup \mathscr{P}_{M}^{*}$; yet if $m(j)>M$ our definitions give $z_{n(m(j))}^{k(j)} \in X \backslash \cup \mathscr{P}_{M}^{*}$, a contradiction. So indeed $Z$ is eventually in int $\cup \mathscr{P}_{M}^{*}$, for some $N \in \mathbf{N}$.

Proposition 5. A Fréchet space $X$ with a $\sigma$-hereditarily closure-preserving $k$ network $\mathscr{P}=\bigcup_{n \in \mathbf{N}} \mathscr{P}_{n}$ is a Lašnev space.

Proof. We assume that $\mathscr{P}_{n} \subset \mathscr{P}_{n+1}$ and, by Lemma 2, that each $\mathscr{P}_{n}$ is closed under finite intersections. For $P \in \mathscr{P}_{n}$ we define

$$
R_{n}(P)=P \backslash \operatorname{int} \bigcup\left\{Q \in \mathscr{P}_{n}: P \not \subset Q\right\}
$$


and

$$
\mathscr{R}_{n}=\left\{R_{n}(P): P \in \mathscr{P}_{n}\right\} .
$$

Claim 5.1. Suppose $Z=\left\{z_{n}: n \in \mathbf{N}\right\}$ converges to $x \in X \backslash Z$ and $N \in \mathbf{N}$. Let $\mathscr{R}^{*}=\left\{R \in \mathscr{R}_{N}: R \cap Z\right.$ is infinite $\}$. If $U$ is an open neighborhood of $x$ and $Z$ is eventually in int $\bigcup\left\{P \in \mathscr{P}_{N}: P \subset U\right\}$, then $Z$ is eventually in int $\cup \mathscr{R}^{*}$ and $\cup \mathscr{R}^{*} \subset$ $U$.

Proof. By Lemma 3, we can find $M$ large enough that $\left\{z_{n}: n \geqslant M\right\} \subset V$, where

$$
V=\operatorname{int} \bigcup \mathscr{P}_{N} \backslash \bigcup\left\{Q \in \mathscr{P}_{N} \cup \mathscr{R}_{N}: Q \cap Z \text { is finite }\right\} .
$$

We show that $V \subset \cup \mathscr{R}^{*}$. If $y \in V$, then $y \in Q \in \mathscr{P}_{N}$ implies $Q \cap Z$ is infinite; thus Lemma 3 gives that $\mathscr{P}_{N}$ is point-finite at $y$ so that $P(y)=\bigcap\left\{Q \in \mathscr{P}_{N}: y \in Q\right\} \in \mathscr{P}_{N}$. Moreover, $y \in X \backslash \cup\left\{Q \in \mathscr{P}_{N}: P(y) \not \subset Q\right\}$, so $y \in R_{N}(P(y))$. Because $y \in V$, we have $R_{N}(P(y)) \cap Z$ is infinite, yielding $y \in R_{N}(P(y)) \subset \cup \mathscr{R}^{*}$, as desired.

To see that $\cup \mathscr{R}^{*} \subset U$, note that if $R_{N}(P) \in \mathscr{R}^{*}$, then $R_{N}(P) \subset P \subset Q$ for some $Q \in \mathscr{P}_{N}$ such that $Q \subset U$ : otherwise $Z$ is eventually in int $\bigcup\left\{Q \in \mathscr{P}_{N}: Q \subset U\right\} \subset$ int $\cup\left\{Q \in \mathscr{P}_{N}: P \not \subset Q\right\} \subset X \backslash R_{N}(P)$, contradicting that $Z \cap R_{N}(P)$ is infinite. This establishes Claim 5.1.

For each $n \in \mathbf{N}$, let $\mathscr{R}_{n}^{\prime}=\mathscr{R}_{n} \cup\left\{X \backslash\right.$ int $\left.\cup \mathscr{R}_{n}\right\}=\left\{R_{\alpha}: \alpha \in I_{n}\right\}$. Let us say that a collection $\mathscr{N}$ of subsets of $X$ forms a net at $x \in X$ if $x \in \mathcal{N}$ and every neighborhood of $x$ contains a member of $\mathscr{N}$. Now let

$$
M=\left\{\sigma \in \prod_{n \in \mathbf{N}} I_{n}:\left\{R_{\sigma(n)}: n \in \mathbf{N}\right\} \text { forms a net at some } x \in X\right\}
$$

and give $M$ the subspace topology inherited from the usual product topology of the discrete spaces $I_{n}$. We define $f: M \rightarrow X$ by $f(\sigma)=x$ if and only if $\left\{R_{\sigma(n)}: n \in \mathbf{N}\right\}$ forms a net at $x$.

Claim 5.2. $f[M]=X$.

Proof. If $x$ is an isolated point, then $\{x\} \in \mathscr{P}_{n}$ for some $n$, and $R_{n}(\{x\})=\{x\}$. If $x$ is not isolated, let $Z$ be a sequence in $X \backslash\{x\}$ converging to $x$. For each $n \in \mathbf{N}$, pick an $R_{\sigma(n)} \in \mathscr{R}_{n}$ so that $R_{\sigma(n)} \cap Z$ is infinite if it is possible to do so. If it is impossible, let $R_{\sigma(n)}=X \backslash$ int $\bigcup \mathscr{R}_{n}$. In any case, $x \in R_{\sigma(n)}$ by Lemma 3 . Then by Lemma 4 and Claim 5.1, $\left\{R_{\sigma(n)}: n \in \mathbf{N}\right\}$ forms a net at $x$.

Claim 5.3. $f$ is continuous.

Proof. Let $U$ be open in $X, x \in U$, and $\sigma \in f^{-1}(x)$. Necessarily there is an $n \in \mathbf{N}$ so that $R_{\sigma(n)} \subset U$ and thus $f[\{\tau \in M: \tau \uparrow n=\sigma \uparrow n\}] \subset R_{\sigma(n)} \subset U$.

Claim 5.4. $f$ is closed.

Proof. Suppose $F$ is a closed subset of $M$ and $Z=\left\{z_{n}: n \in \mathbf{N}\right\}$ is a sequence in $f(F)$ converging to $x \in X \backslash Z$. For each $n \in \mathbf{N}$, select a $\sigma_{n} \in F \cap f^{-1}\left(z_{n}\right)$. Let $S_{0}=\mathbf{N}$, and for every $m \in \mathbf{N}$ we inductively choose an infinite $S_{m} \subset S_{m-1}$ and $\tau(m) \in I_{m}$ as follows.

By Lemma 3, find an $M \in \mathbf{N}$ so that $\mathscr{R}^{*}=\left\{R \in \mathscr{R}_{m}^{\prime}: R \cap Z\right.$ is infinite $\}=\{R \in$ $\left.\mathscr{R}_{m}^{\prime}: R \cap\left\{z_{n}: n \geqslant M\right\} \neq \varnothing\right\}$ is finite. So for $n \geqslant M, R_{\sigma_{n}(m)} \in \mathscr{R}^{*} ;$ as a result there is an infinite $S_{m} \subset S_{m-1}$ so that the $\sigma_{n}(m)$ 's $\left(n \in S_{m}\right)$ coincide, say $\tau(m)=\sigma_{n}(m)$ for all $n \in S_{m}$. 
We show that the $\tau \in \Pi_{m \in \mathbf{N}} I_{m}$ thus chosen is in $f^{-1}(x)$. Since for each $m \in \mathbf{N}$, $z_{n} \in R_{\sigma_{n}(m)}=R_{\tau(m)}$ for all $n \in S_{m}$, we have $x \in \bigcap_{m \in \mathrm{N}} R_{\tau(m)}$. If $U$ is an open set with $x \in U$, then Lemma 4 and Claim 5.1 produce an $N \in \mathbf{N}$ so that $R_{\tau(N)} \in \mathscr{R}_{N}$ and $R_{\tau(N)} \subset U$. Thus $f(\tau)=x$.

If we pick $n(m) \in S_{m}, n(m)<n(m+1)$ for each $m \in \mathbf{N}$, then $\left\{\sigma_{n(m)}: m \in \mathbf{N}\right\}$ converges to $\tau$. Indeed, if $m \geqslant k$, then $n(m) \in S_{k}$, so $\sigma_{n(m)}(k)=\tau(k)$. Thus $\tau \in F$, $x \in f(F)$, and $f(F)$ is closed.

We give a new proof for the following unpublished result.

Corollary 6 [Guthrie]. A Hausdorff space is metrizable if and only if it is first countable and has a $\sigma$-hereditarily closure-preserving $k$-network.

Proof. By a theorem of Morita, Hanai [9] and Stone [11], a first countable Lašnev space is metrizable.

COROLlaRy 7 [10]. A Hausdorff space is metrizable if and only if it is first countable and has a $\sigma$-locally finite $k$-network.

COROLlaRy 8 [2]. A regular space is metrizable if and only if it has a $\sigma$-hereditarily closure-preserving base.

Proof. By [2, Lemma 4] a space with a $\sigma$-hereditarily closure-preserving base is first countable.

The next corollary answers a question of [12].

Corollary 9. A Hausdorff Fréchet space with a countable k-network is a Lašnev space.

Lašnev [6] characterized closed images of metric spaces as $T_{1}$ Fréchet spaces with an almost refining sequence of hereditarily closure-preserving coverings comprising a network for the space. (See [6] for the definition.) Although Lašnev's proof and that which we have given here are quite similar, the two characterizations are different. Lašnev's proof of the sufficiency works if "Fréchet space" is replaced by " $k$-space," while there is a regular $k$-space with a $\sigma$-discrete $k$-network which is not normal [3].

\section{REFERENCES}

1. A. Arhangel'ski1̄, Some types of factor mappings and the relations between classes of topological spaces, Soviet Math. Dokl. 4 (1963), 1726-1729.

2. D. Burke, R. Engelking and D. Lutzer, Hereditarily closure-preseving collections and metrization, Proc. Amer. Math. Soc. 51 (1975), 483-488.

3. L. Foged, Normality in $k$ - and $\aleph$-spaces, Topology Appl. (to appear).

4. S. Franklin, Spaces in which sequences suffice, Fund. Math. 57 (1965), 107-115.

5. G. Gruenhage, E. Michael and Y. Tanaka, Spaces determined by point-countable covers, Pacific J. Math. 113 (1984), 303-332.

6. N. Lašnev, Closed images of metric spaces, Soviet Math. Dokl. 7 (1966), 1219-1221.

7. E. Michael, A note on closed maps and compact sets, Israel J. Math. 2 (1964), 173-176.

8. $\boldsymbol{\aleph}_{0}$-spaces, J. Math. Mech. 15 (1966), 983-1002.

9. K. Morita and S. Hanai, Closed mappings and metric spaces, Proc. Japan Acad. 32 (1956), 10-14.

10. P. O'Meara, A metrization theorem, Math. Nachr. 45 (1970), 69-72.

11. A. Stone, Metrizability of decomposition spaces, Proc. Amer. Math. Soc. 7 (1956), 690-700.

12. Y. Tanaka, Closed images of locally compact spaces and Fréchet spaces, Topology Proc. 7 (1982), $279-292$.

Department of Mathematics, University of Texas at El Paso, El Paso, Texas 79968 